\title{
The Discriminating Power of Items That Measure More Than One Dimension
}

\author{
Mark D. Reckase, American College Testing \\ Robert L. McKinley, Educational Testing Service
}

Determining a correct response to many test items frequently requires more than one ability. This paper describes the characteristics of items of this type by proposing generalizations of the item response theory concepts of discrimination and information. The conceptual framework for these statistics is presented, and the formulas for the statistics are derived for the multidimensional extension of the two-parameter logistic model. Use of the statistics is demonstrated for a form of the ACT Mathematics Usage Test. Index terms: item discrimination, item information, item response theory, multidimensional item response theory.

Statistical measures of item discrimination are computed for several reasons. The traditional measures, usually the point-biserial or biserial correlations between item scores and total scores, are used as general indicators of item quality or as screening variables for use in selecting items for a test. During test construction, items are often selected that have a discrimination index that is greater than a specified value, such as .20 or .30 . Discrimination indices may also be used to determine whether an item measures the same construct as the total score on the test. For the most part, the item response theory (IRT) measures of discrimination-and the related concept of item information-are used in these same ways, but they are also used to specify the precision of measurement provided by an item at different levels of ability $(\theta)$ along the $\theta$ scale.

Both the traditional and IRT measures of the discriminating power of an item are based on the assumption that a test measures a single trait-either that defined by the total score or by the $\theta$ scale. This paper generalizes the concept of item discrimination to the case in which more than one ability is required to determine the correct response to an item. In making this generalization, the conceptual framework supplied by IRT is used. This paper also draws on the definition of multidimensional item difficulty (MID) developed by Reckase (1985).

In unidimensional IRT, the difficulty of an item is indicated by the point on the $\theta$ scale where the slope of the item response function (IRF) is the steepest, which is the same point as the point of inflection of the IRF. MID, similarly, is the point where the item response surface (IRS) (i.e., the probability of a correct response for each point in the multidimensional $\theta$ space) is steepest when the slope is determined in the direction from the origin of the space. This is also a point of inflection for the IRS. Thus, MID indicates the location in the $\boldsymbol{\theta}$ space where the test item is most discriminating.

In this paper, multidimensional discrimination (MDISC) and multidimensional information (MINF) are defined. The definitions are consistent with current IRT methodology and are helpful in understanding how an item is functioning when assessing multiple skills. To assist in the use of these item statistics, the concepts are presented in three ways: (1) a conceptual framework is developed; (2) the general concepts are applied to a particular multidimensional item response theory (MIRT) model, and the MDISC and MINF statistics are derived for that model; and (3) MDISC and MINF are computed 
using item response data from a form of the American College Testing (ACT) Mathematics Usage Test. The interpretation and use of the statistics are also discussed.

\section{Conceptual Framework}

More than one ability is often necessary to respond correctly to test questions. For example, reading, problem solving, and computational abilities may be needed to solve mathematics story problems. In most IRT models, it is assumed that the probability of a correct response to an item increases with an increase in the level of the trait being measured. Likewise in MIRT models, it is assumed that the probability of a correct response increases when there is an increase in any one-or any combinationof the abilities required for solving the item. Therefore, in one region of the $\theta$ space the probability of a correct response will be very high, whereas in another region of the $\theta$ space, the probability will be very low. In general, the discriminating power of an item indicates how quickly the transition takes place from low probability to high probability of a correct response. A highly discriminating item divides the regions clearly-having a narrow region of ambiguity, that is, a region where the probabilities are intermediate in magnitude. Figure 1 shows the IRSS for two items, one (Item 1) with moderately high discrimination and the other (Item 2) with lower discrimination. Note that the two items do not discriminate in the same direction in the $\theta$ space.

The measures of discrimination proposed here describe the characteristics of an item in two ways. First, a measure of the maximum level of item discrimination is presented. Item discrimination is related to the slope of the IRS. The slope differs, however, depending on the location in the $\boldsymbol{\theta}$ space and the direction relative to the surface at that location. For example, for Item 1 in Figure la, the slope is almost flat at the point $(-2,-2)$ in any direction; at the point $(2,-3)$ the slope is steep in a direction roughly parallel to $\theta_{1}$; and it is fairly low, or even zero, in a direction that travels across the surface. The maximum level of item discrimination is at a location and in a direction where the slope is steepest. The steeper the slope, the more clearly the item divides the $\theta$ space into two regions. Clearly, Item 1 in Figure 1 more clearly divides the $\boldsymbol{\theta}$ space into two regions than does Item 2 . Item 1 is more discriminating than Item 2 overall, but they are not dividing the $\boldsymbol{\theta}$ space in the same way.

The discriminating power of an item can also be described relative to a particular direction in the $\theta$ space. Using this measure, two items can be compared directly to determine which is a better measure of a particular trait. In Figure 1, for example, Item 2 is better than Item 1 at differentiating two persons who are at points $\left(\theta_{1}=1, \theta_{2}=0\right)$ and $\left(\theta_{1}=1, \theta_{2}=1\right)$ in the $\theta$ space. Note that these two persons only differ on $\theta_{2}$. Thus, Item 2 has more MINF for measuring $\theta_{2}$ in the specified region of the $\theta$ space. In fact, Item 2 has a faster rate of change in probabilities than Item 1 when the rate is determined in a direction parallel to $\theta_{2}$.

\section{Theoretical Framework}

The work presented below is based on the assumption that the interaction between a person and an item can be described by one of a class of well-behaved probability functions. These functions relate the probability of a correct item response to a person's location in a multidimensional $\boldsymbol{\theta}$ space, as indicated by the ability vector $\boldsymbol{\theta}$, and the characteristics of the item, as indicated by a vector of item parameters, $\sigma$. That is,

$P\left(x_{i j}=1\right)=f\left(\sigma_{i}, \theta_{j}\right)$,

where $x_{i j}$ is the score on item $i$ for person $j$. This function is assumed to be "well behaved" because for all dimensions in the space, or any combination of dimensions, as $\theta_{j}$ increases, $P\left(x_{i j}=1\right)$ is nondecreasing. 
Figure 1

Response Surfaces for Items That Vary in Discriminating Power and Dimension Assessed

a. Item 1

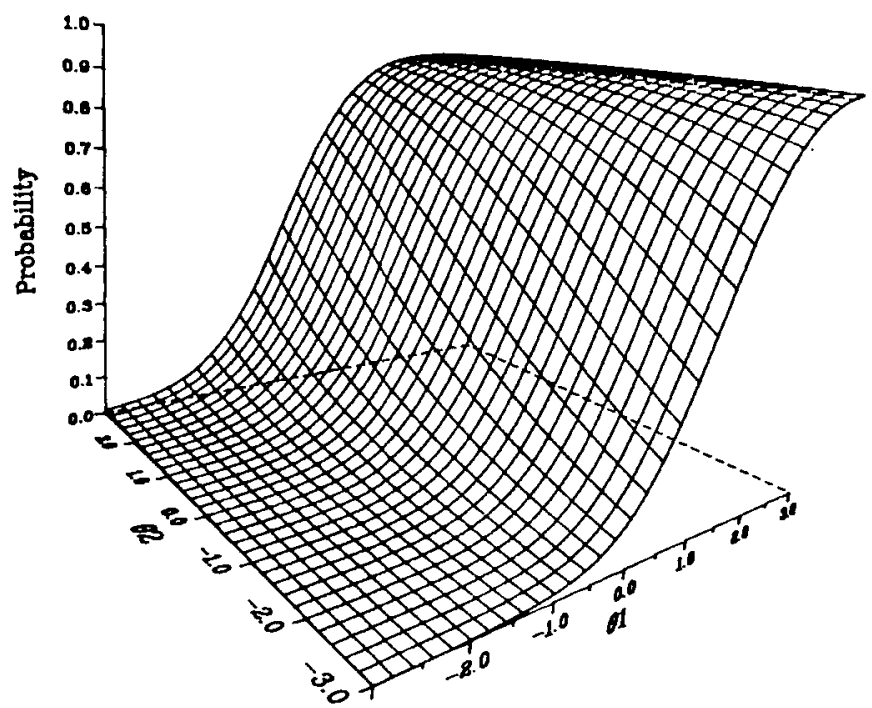

b. Item 2

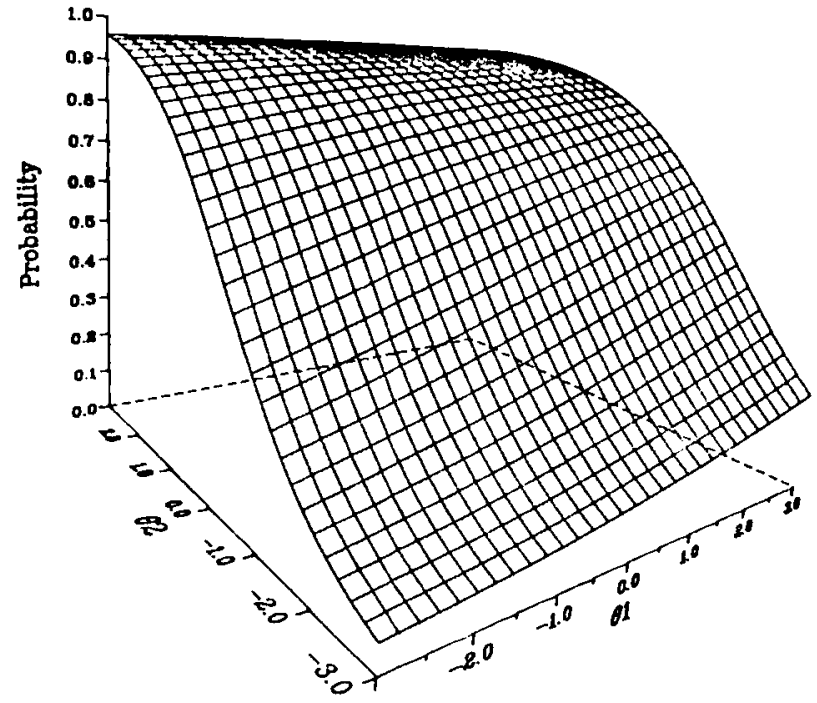

\section{Multidimensional Discrimination}

A measure of item discrimination for an item that can be described by Equation 1 is useful to the extent that it provides the same type of information that is provided by the unidimensional discrimination statistics. That is, MDISC should allow items to be compared on a general measure of quality, to be classified as above or below a standard of quality, and to be used as an indicator of strength of the relationship of item performance for each dimension in the $\theta$ space. Further, it 
would be especially convenient if the MDISC statistics were related to the MID statistics in the same way that unidimensional IRT item statistics are related, because then some of the interpretive framework that has been developed for the unidimensional case could be generalized to the multidimensional case.

In unidimensional IRT, the discrimination parameter is related to the slope of the IRF at the point where the slope is steepest, the point of inflection. The point of inflection is also used to define the difficulty parameter for an item. The most direct generalization of this concept to the multidimensional case would be to relate MDISC to the slope of the surface defined by Equation 1 at the point of steepest slope on the surface in the multidimensional space.

This definition of MDISC, however, has several problems. For some mathematical forms of the function in Equation 1 (e.g., Sympson, 1978), the point of maximum slope occurs when at least one of the $\theta$ s approaches infinity. Therefore, the MDISC statistics could not be related to MID in the same way that is true for the unidimensional models. For other classes of models (e.g., Equation 5), there are an infinite number of points of maximum slope. This fact also causes difficulties when interpreting the MDISC statistic. Therefore, an alternative definition for MDISC is proposed that uniquely determines a single value for MDISC and closely relates it to the MID.

The proposed definition specifies the MDISC as a function of the slope of the IRS defined by Equation 1 at the steepest point in the direction indicated by the MID. This is the direction from the origin of the $\theta$ space in which the IRS has its steepest slope. Conceptually, determining the MDISC value requires several steps. First, a direction from the origin of the space is selected, and the point of inflection of the IRS along the line defined by this direction is determined. This process determines the point of steepest slope in the direction specified. Next, the slope at the point of inflection in the specified direction is determined. The same process is followed in each direction from the origin, and the steepest slopes are determined. These slopes are compared to determine the steepest slope, which then is used to compute the MDISC statistic. The direction that gives the steepest slope is the same as the direction specified by the MID. The distance from the origin to the point of steepest slope in the direction indicated by the MID is the distance component of the MID statistic.

The MDISC statistic is an overall measure of the capability of an item to distinguish between individuals that are in different locations in the $\boldsymbol{\theta}$ space. A similar statistic can also be developed that is conditional on any particular direction in the space. An interesting special case would be to determine the discrimination in directions along the coordinate axes. These conditional statistics would give information about how well the item measures a particular dimension.

The mathematical procedure for determining MDISC has four steps.

1. To simplify the analysis, first convert the mathematical expression for the IRS to polar coordinates.

2. Use the second derivative in direction $\alpha$ from the origin to determine the point of steepest slope in that direction.

3. Determine the expression for the slope at the point of steepest slope using the first derivative.

4. Take the first derivative with respect to $\alpha$ to determine the direction of overall steepest slope.

A function of the slope in that direction is proposed as the MDISC statistic.

This procedure yields a value of MDISC that has the same relationship to MID that the $a$ parameter has to the $b$ parameter in unidimensional IRT. This relationship is demonstrated below when the MDISC definition is applied to a particular MIRT model.

\section{Multidimensional Information}

MINF is related to the MDISC in that if an item has a high value of MDISC, it will provide a large amount of information somewhere in the $\theta$ space. However, MINF differs from MDISC because MINF 
is concerned with the capability of the item to discriminate at each point in the space, rather than just at the steepest point of the IRS.

The definition of MINF proposed here is a direct generalization of the unidimensional IRT concept of information. In unidimensional IRT, information at a $\theta$ level is the ratio of the square of the slope of the IRF at $\theta$ to the variance of error of the item score at that level of $\theta$. Mathematically, item information is expressed as

$I_{i}(\theta)=\frac{\left(\frac{\delta P_{i}(\theta)}{\delta \theta}\right)^{2}}{P_{i}(\theta) Q_{i}(\theta)}$,

where $I_{i}(\theta)$ is the information from item $i$ at $\theta, P_{i}(\theta)$ is the probability of a correct response to item $i$ for a person with ability $\theta$, and $Q_{i}(\theta)=1-P_{i}(\theta)$. Test information is simply the sum of item information values, or

$I_{T}(\theta)=\sum_{i=1}^{n} I_{i}(\theta)$,

where $n$ is the number of items.

For the multidimensional case, Equations 2 and 3 can still be used, but the slope in the numerator of Equation 2 must be determined in a slightly different way. For an IRS, there are many slopes at any point in the $\boldsymbol{\theta}$ space rather than one. Depending on the direction that is taken at the point in the space, the slope will differ. The slope will be much greater if a direction is selected that goes up the surface rather than one that goes across it. One direction may yield a slope of 0 ; but another direction, at the same point in the space, may yield a fairly steep slope. Thus, direction in the space must be considered when determining the information provided by the item. This is the same as specifying how much information is provided about a particular composite of abilities at a point in the $\boldsymbol{\theta}$ space.

To determine the slope in a particular direction, the mathematical procedure known as the directional derivative is needed. The directional derivative is defined as

$\nabla_{\alpha} P(\boldsymbol{\theta})=\frac{\delta P(\boldsymbol{\theta})}{\delta \theta_{1}} \cos \alpha_{1}+\frac{\delta P(\boldsymbol{\theta})}{\delta \theta_{2}} \cos \alpha_{2}+, \ldots,+\frac{\delta P(\boldsymbol{\theta})}{\delta \theta_{n}} \cos \alpha_{n}$,

where $\boldsymbol{\alpha}$ is the vector of angles with the coordinate axes in the $\theta$ space,

$\alpha_{i}(i=1, \ldots, n)$ is an element of the vector,

$\boldsymbol{\theta}$ is the vector of abilities defining a point in the space, and

$\theta_{i}(i=1, \ldots, n)$ is an element of the vector.

Equation 4 gives the slope in direction $\boldsymbol{\alpha}$ at the point $\boldsymbol{\theta}$ in the $\boldsymbol{\theta}$ space.

When computing MINF, the directional derivative replaces the derivative in the numerator of Equation 2. Then item information can be determined for any angle-each angle representing different composites of abilities-in the space. Thus, to totally describe the information structure of an item, many information plots are needed. In principal, an information function can be determined for the infinite number of directions from the origin. In practice, determining the information function for angles at $10^{\circ}$ intervals between $0^{\circ}$ and $90^{\circ}$ from the axes is sufficient to determine where, and for what combination of abilities, an item provides information.

\section{MDISC and MINF for the Multidimensional Extension of the Two-Parameter Logistic Model (M2PL)}

To demonstrate the use of MDISC and MINF, a MIRT model is needed that can be used to derive 
the mathematical expressions for the statistics. Reckase (1985) developed the MID concept using the M2PL model, and an estimation program is available for the model (McKinley \& Reckase, 1983); therefore, the M2PL model is used here as an example. However, the concepts can also be applied to other MIRT models.

The M2PL model is given by

$$
P\left(x_{i j}=1 \mid \mathbf{a}_{i}, d_{i}, \boldsymbol{\theta}_{j}\right)=\frac{\exp \left(\mathbf{a}_{i}^{\prime} \boldsymbol{\theta}_{j}+d_{i}\right)}{1+\exp \left(\mathbf{a}_{i}^{\prime} \boldsymbol{\theta}_{j}+d_{i}\right)},
$$

where $x_{i j}$ is the score $(0,1)$ on item $i$ by person $j$,

$\mathbf{a}_{i}$ is the vector of item discrimination parameters,

$d_{i}$ is a scalar parameter that is related to the difficulty of the item, and

$\boldsymbol{\theta}_{j}$ is the vector of ability parameters for person $j$.

\section{Multidimensional Discrimination}

The MDISC for an item is a function of the slope at the steepest point in the MID direction for an item. Reckase (1985) derived the MID direction as

$$
\cos \alpha_{i k}=\frac{a_{i k}}{\left(\sum_{k=1}^{m} a_{i k}^{2}\right)^{1 / 2}}, \quad k=1, \ldots, m,
$$

where $\alpha_{i k}$ is the angle with axis $k$ for item $i$,

$a_{i k}$ is the $k$ th element of vector $\mathbf{a}_{i}$, and

$m$ is the number of dimensions in the space.

He also determined that the slope of the IRS at the point of inflection in direction $\boldsymbol{\alpha}_{i}$ is

Slope $=\frac{1}{4} \sum_{k=1}^{m} a_{i k} \cos \alpha_{i k}$

Substituting Equation 6 into Equation 7 yields the slope in the MID direction:

Slope $=\frac{1}{4} \sum_{k=1}^{m} a_{i k} \frac{a_{i k}}{\left(\sum_{k=1}^{m} a_{i k}^{2}\right)^{1 / 2}}=\frac{1}{4}\left(\sum_{k=1}^{m} a_{i k}^{2}\right)^{1 / 2}$.

For the unidimensional two-parameter logistic (2PL) model, the slope at the point of inflection is equal to $(1 / 4) a_{i}$.

Thus, $\left(\sum_{k=1}^{m} a_{i k}^{2}\right)^{1 / 2}$ is analogous to the $a$ parameter in the unidimensional model. Therefore, the MDISC can be defined as

$M D I S C=\left(\sum_{k=1}^{m} a_{i k}^{2}\right)^{1 / 2}$

This definition of MDISC has several useful properties. First, if an item measures only dimension $\ell$, that is, when $a_{i \ell}>0$ and $a_{i j}=0$ for all $j \neq \ell$, MDISC $=a_{i \ell}$. Thus, for this special case, the MDISC is equal to the unidimensional discrimination parameter (as it should be).

Second, when the 2PL model is expressed in the slope-intercept form, the exponent is given by $a_{i} \theta_{j}+d_{i}$, where $d_{i}=-b_{i} a_{i}$. The distance, $D_{i}$, in the MID has the same relationship with the inter- 
cept term, $d_{i}$, of Equation 5 as $b_{i}$ does with $d_{i}$ for the 2PL model. That is,

$d_{i}=-D_{i} \cdot M D I S C$,

because

$D_{i}=\frac{-d_{i}}{\left(\sum_{k=1}^{m} a_{i k}^{2}\right)^{1 / 2}}=\frac{-d_{i}}{M D I S C}$.

Finally, MDISC is on the same scale as $a_{i k}$-four times the slope-so it can be interpreted accordingly. Therefore, the definition meets all the requirements stated above for a generalization of the IRT discrimination parameter.

\section{Multidimensional Information}

To compute the MINF for the M2PL model, the directional derivative of the IRS is needed. The directional derivative is given by

$$
\begin{aligned}
\nabla_{\alpha} P_{i}(\boldsymbol{\theta}) & =a_{i 1} P_{i}(\boldsymbol{\theta}) Q_{i}(\boldsymbol{\theta}) \cos \alpha_{1}+a_{i 2} P_{i}(\boldsymbol{\theta}) Q_{i}(\theta) \cos \alpha_{2}+, \ldots,+a_{i m} P_{i}(\boldsymbol{\theta}) Q_{i}(\theta) \cos \alpha_{m} \\
& =P_{i}(\boldsymbol{\theta}) Q_{i}(\boldsymbol{\theta}) \sum_{k=1}^{m} a_{i k} \cos \alpha_{k} .
\end{aligned}
$$

This expression can be substituted for the term in the numerator of Equation 2, yielding

$$
\begin{aligned}
I_{\alpha}(\theta) & =\frac{\left[P_{i}(\theta) Q_{i}(\theta) \sum_{k=1}^{m} a_{i k} \cos \alpha_{k}\right]^{2}}{P_{i}(\theta) Q_{i}(\theta)} \\
& =P_{i}(\theta) Q_{i}(\theta)\left(\sum_{k=1}^{m} a_{i k} \cos \alpha_{k}\right)^{2} .
\end{aligned}
$$

From this equation, the information at the point indicated by $\theta$ in direction $\alpha$ can be determined. As with the unidimensional definition of information, the item information functions can be summed to obtain a test information function. However, when test information is computed, the same direction must be used for all the items.

\section{Example of the Application of MDISC and MINF}

To demonstrate the use of MDISC and MINF, Form 24B of the ACT Mathematics Usage Test (American College Testing, 1982) was analyzed to determine estimates of the parameters of the M2PL model. Responses from a systematic sample of 1,000 examinees were used. The MAXLOG program (McKinley \& Reckase, 1983) was used to estimate the parameters. A two-dimensional solution was obtained so that the results could be represented graphically.

\section{MDISC and MINF Estimates for All Items}

The parameter estimates for the M2PL model, the MID (both the direction and the distance), and the MDISC statistics for the 40 items in the test are presented in Table 1 . Item 27 had the highest MDISC statistic, which means that this item was the best at differentiating between examinees in different parts in the $\theta$ space. However, this item discriminated best along a line that is at a $46^{\circ}$ angle to the Dimension 1 axis. Along the Dimension 1 axis (at $0^{\circ}$ to the axis), the discrimination was only 1.66 , 
n.: Table 1

Item Parameters, MID, and MDISC for the Items in the ACT Mathematics Usage Test, Form 24B

\begin{tabular}{|c|c|c|c|c|c|c|c|}
\hline \multirow[b]{2}{*}{ Item } & \multirow[b]{2}{*}{$a_{11}$} & \multirow[b]{2}{*}{$a_{12}$} & \multirow[b]{2}{*}{$d_{i}$} & \multicolumn{3}{|c|}{ MID } & \multirow[b]{2}{*}{ MDISC } \\
\hline & & & & $\alpha_{i 1}$ & $\alpha_{i 2}$ & $D_{t}$ & \\
\hline 1 & 1.81 & .86 & 1.46 & 25 & 65 & -.73 & 2.00 \\
\hline 2 & 1.22 & .07 & .17 & 4 & 89 & -.14 & 1.22 \\
\hline 3 & 1.57 & .36 & .67 & 13 & 77 & -.42 & 1.61 \\
\hline 4 & .71 & .53 & .44 & 37 & 53 & -.50 & .89 \\
\hline 5 & .86 & .19 & .10 & 12 & 78 & -.11 & .88 \\
\hline 6 & 1.72 & .18 & .44 & 6 & 84 & -.25 & 1.73 \\
\hline 7 & 1.86 & .29 & .38 & 9 & 81 & -.20 & 1.88 \\
\hline 8 & 1.33 & .34 & .69 & 14 & 76 & -.50 & 1.37 \\
\hline 9 & 1.19 & 1.57 & .17 & 53 & 37 & -.09 & 1.97 \\
\hline 10 & 2.00 & 0.00 & .38 & 0 & 90 & -.19 & 2.00 \\
\hline 11 & .87 & 0.00 & .03 & 0 & 90 & -.03 & .87 \\
\hline 12 & 2.00 & .98 & .91 & 26 & 64 & -.41 & 2.23 \\
\hline 13 & 1.00 & .89 & -.49 & 42 & 48 & .37 & 1.34 \\
\hline 14 & 1.22 & .14 & .54 & 7 & 83 & -.44 & 1.23 \\
\hline 15 & 1.27 & . 47 & .29 & 20 & 70 & -.21 & 1.35 \\
\hline 16 & 1.35 & 1.15 & -.21 & 40 & 50 & .12 & 1.77 \\
\hline 17 & 1.06 & .45 & .08 & 23 & 67 & -.07 & 1.15 \\
\hline 18 & 1.92 & 0.00 & .12 & 0 & 90 & -.06 & 1.92 \\
\hline 19 & .96 & .22 & -.30 & 13 & 77 & .30 & .98 \\
\hline 20 & 1.20 & .12 & -.28 & 6 & 84 & .23 & 1.21 \\
\hline 21 & 1.41 & .04 & -.21 & 2 & 88 & .15 & 1.41 \\
\hline 22 & 1.54 & 1.79 & .02 & 49 & 41 & -.01 & 2.36 \\
\hline 23 & .54 & .23 & -.69 & 23 & 67 & 1.18 & .59 \\
\hline 24 & 1.53 & .48 & -.83 & 17 & 73 & .52 & 1.60 \\
\hline 25 & .72 & .55 & -.56 & 37 & 53 & .62 & .91 \\
\hline 26 & .51 & .65 & -.49 & 52 & 38 & .59 & .83 \\
\hline 27 & 1.66 & 1.72 & -.38 & 46 & 44 & .16 & 2.39 \\
\hline 28 & .69 & .19 & -.68 & 15 & 75 & .95 & .72 \\
\hline 29 & .88 & 1.12 & -.91 & 52 & 38 & .64 & 1.42 \\
\hline 30 & .68 & 1.21 & -1.08 & 61 & 29 & .78 & 1.39 \\
\hline 31 & .24 & 1.14 & -.95 & 78 & 12 & .82 & 1.36 \\
\hline 32 & .51 & 1.21 & -1.00 & 67 & 23 & .76 & 1.31 \\
\hline 33 & .76 & .59 & -.96 & 38 & 52 & 1.00 & .96 \\
\hline 34 & .01 & 1.94 & -1.92 & 90 & 0 & .99 & 1.94 \\
\hline 35 & .39 & 1.77 & -1.57 & 78 & 12 & .87 & 1.81 \\
\hline 36 & .76 & .99 & -1.36 & 52 & 38 & 1.09 & 1.25 \\
\hline 37 & .49 & 1.10 & -.81 & 66 & 24 & .67 & 1.20 \\
\hline 38 & .29 & 1.10 & -.99 & 75 & 15 & .87 & 1.14 \\
\hline 39 & .48 & 1.00 & -1.56 & 64 & 26 & 1.41 & 1.11 \\
\hline 40 & .42 & .75 & -1.61 & 61 & 29 & 1.87 & .86 \\
\hline
\end{tabular}

which was less than the discrimination for Item 10 along the Dimension 1 axis. Thus, the MDISC gives an overall measure of the quality of the item, but clearly the item is not of equal quality in measuring in all directions (i.e., for all weighted composites of abilities).

The MDISC statistics for two items can only be directly compared if the items measure in the same direction (e.g., Items 29 and 36, for which $\alpha_{29,1}=\alpha_{36,1}=52$ and $\alpha_{29,2}=\alpha_{36,2}=38$ ). The MDISC 
statistics cannot be compared for Items 3 and 30 because the directions are quite different. To compare these items, a common direction $a$ would have to be selected, and then the discrimination in the $\alpha$ direction would have to be computed using the formula

Directional Discrimination $=\sum_{k=1}^{m} a_{i k} \cos \alpha_{i k}$.

For Items 3 and 30, the directional discriminations in direction $30^{\circ}$ from Dimension 1 were 1.54 and 1.19 , respectively; at $60^{\circ}$ from Dimension 1 , they were 1.10 and 1.39 , respectively. Thus, depending on the direction, the ordering of the items on discrimination changes. However, Item 3 was more discriminating overall because it has a higher MDISC statistic.

Table 2 provides correlations among the MDISC statistics, the biserial correlation between the item and the total score on the test $\left(r_{B I S}\right)$, the $a$ parameter estimates from the three-parameter logistic model obtained from LOGIST (Wingersky, Barton, \& Lord, 1982) $\left(a_{\text {LoGIST }}\right)$, and the a parameter estimates from the M2PL model. The MDISC statistic for this dataset had the highest correlation with the $r_{B I S}$ statistic. It is interesting that $a_{L O G I S T}$ is most highly correlated with $a_{2}$, but $r_{B I S}$ is most highly related to $a_{1}$. The relationship between MDISC and $a_{1}$ and $a_{2}$ is dictated by Equation 9 .

Table 2

Correlations Among Discrimination Parameter Estimates

\begin{tabular}{lcccc}
\hline \hline & \multicolumn{4}{c}{ Parameter } \\
\cline { 2 - 5 } Parameter & $a_{\text {LOGIST }}$ & $r_{B I S}$ & $a_{1}$ & $a_{2}$ \\
\hline MDISC & .46 & .78 & .62 & .46 \\
$a_{\text {LOGIST }}$ & & .14 & -.21 & .74 \\
$r_{B I S}$ & & & .80 & .16 \\
$a_{1}$ & & & & -.34 \\
\hline
\end{tabular}

\section{MINF for A Single Item}

The MINF was computed for Item 10 using directions of $0^{\circ}, 30^{\circ}, 60^{\circ}$, and $90^{\circ}$ from Dimension 1 . The results for this item are shown in two different ways in Figures 2 and 3. Figure 2 indicates the amount of information in direction $\boldsymbol{\alpha}$ by the height of the surface above the $\theta$ plane. The four parts of Figure 2 show the surfaces for each of the four directions. The figures show that the item gives no information about $\theta_{2}$, and that the amount of information provided by the item increases as the angle decreases from $90^{\circ}$ (Figure $2 \mathrm{~d}$ ) to $0^{\circ}$ (Figure $2 \mathrm{a}$ ). In all cases, the information is greatest along the line, $\theta_{1}=-.19$.

These same data are presented in Figure 3 using a representation scheme suggested by Thissen (personal communication, 1984). At selected points in the $\theta$ space, the information is represented by the length of the line in the direction taken in the space. Lines are given at $10^{\circ}$ intervals. Figure 3 shows that Item 10 gives no information about Dimension 2, but progressively provides more information as the angle decreases from $90^{\circ}$ to $0^{\circ}$ with respect to Dimension 1. Most of the information provided in the four parts of Figure 2 is given in Figure 3.

\section{Test Information}

The information supplied by the entire test is shown by the three surfaces in Figure 4 and the line plots in Figure 5. Comparison of Figures $4 \mathrm{a}$ and $4 \mathrm{c}$ shows that the test supplies somewhat more information about $\theta_{1}$ than $\theta_{2}$. Figure $4 \mathrm{~b}$ shows that the most information is provided when 

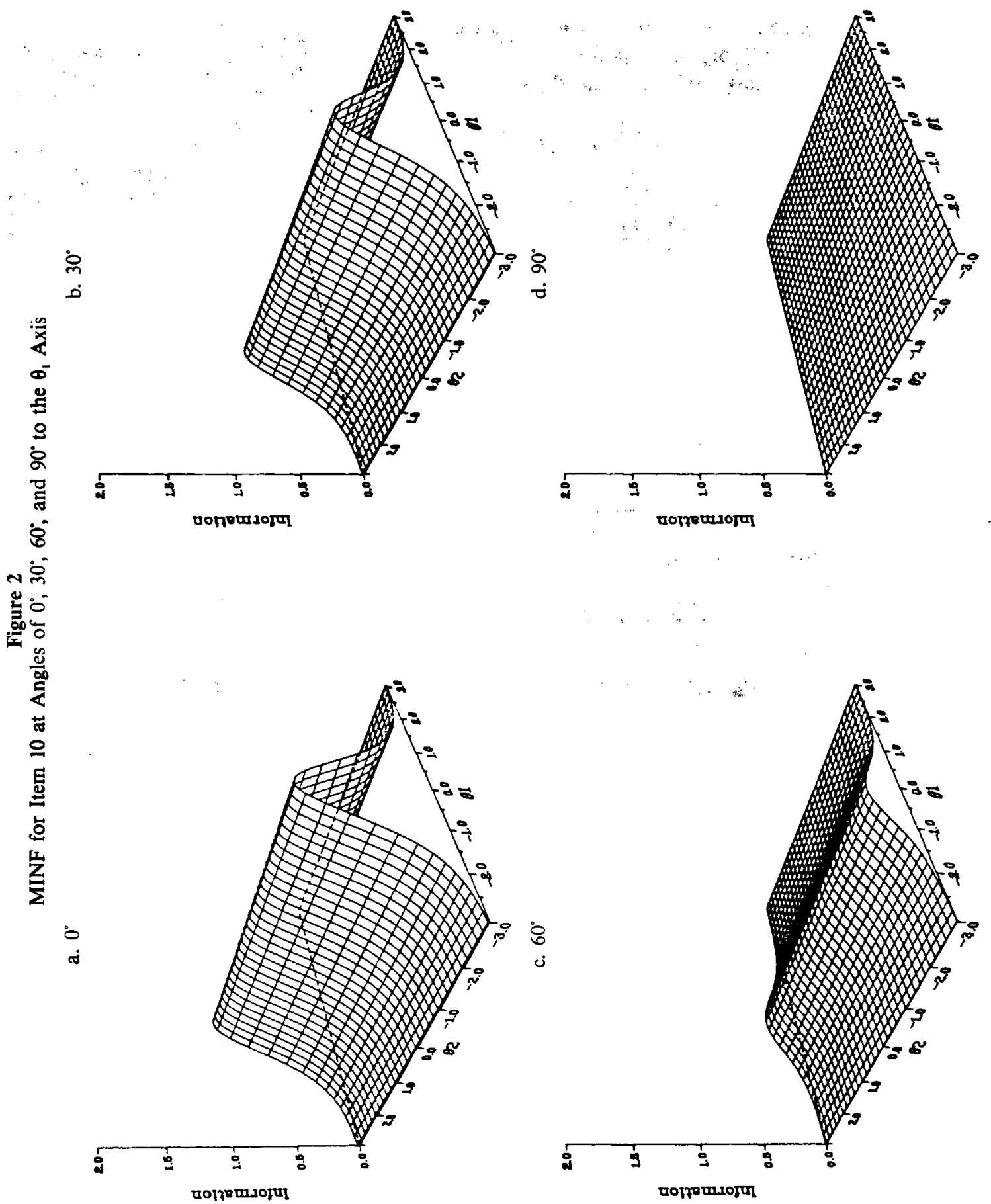

N

象。

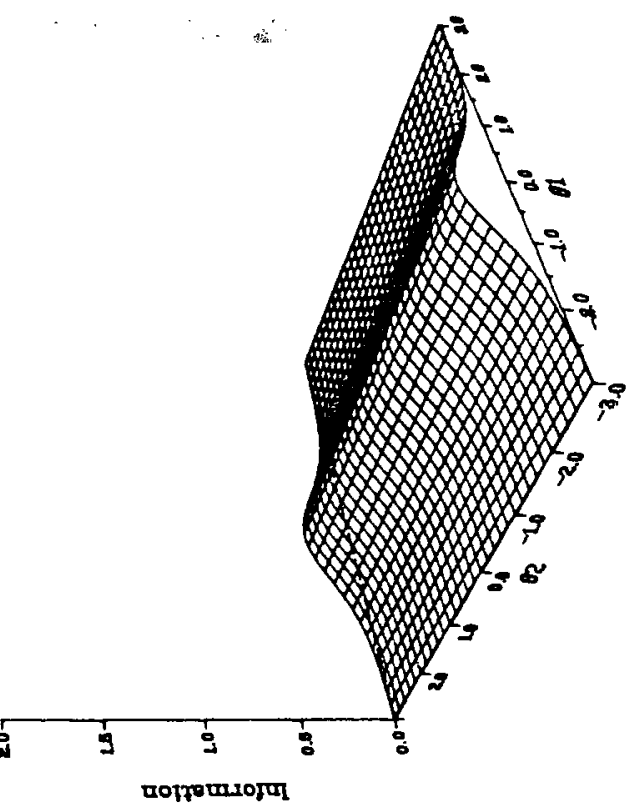


Figure 3

Information for Item 10 Represented by Directional Vectors

From Points in the $\theta$ Space

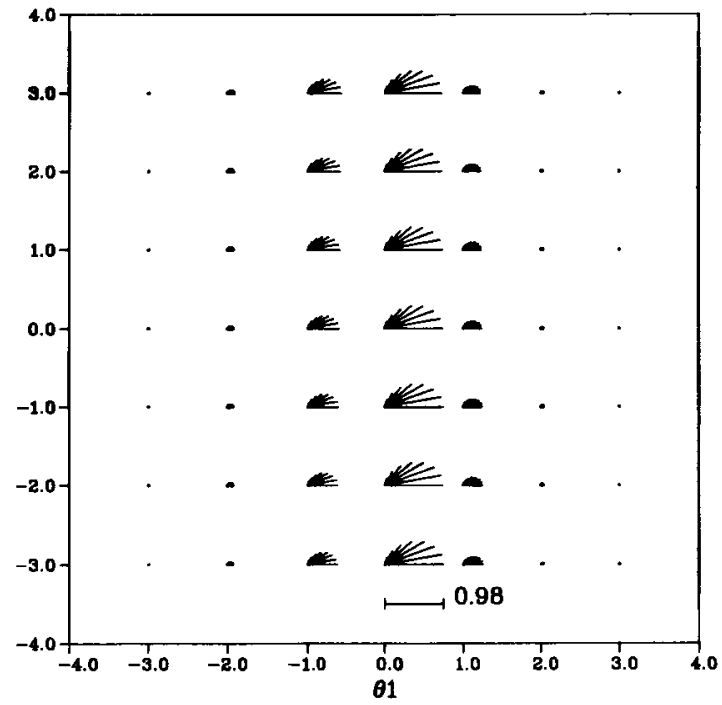

the direction is $45^{\circ}$ to $\theta_{1}$; this is equivalent to an equally weighted composite of $\theta_{1}$ and $\theta_{2}$. The same information is given in Figure 5, but the line plot more clearly indicates the regions of the $\theta$ space that are best measured by the test.

\section{Figure 5}

MINF for the ACT Mathematics Usage Test Represented as Directional Vectors From Points in the $\theta$ Space

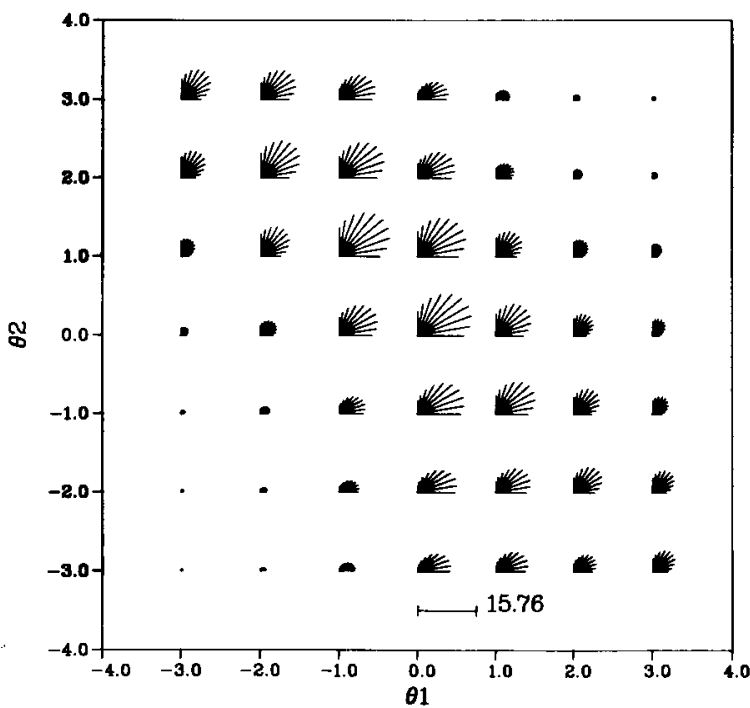


Figure 4

MINF for the ACT Mathematics Usage Test at Angles of $0,45^{\circ}$, and $90^{\circ}$ to the $\theta_{1}$ Axis

a. $0^{\circ}$

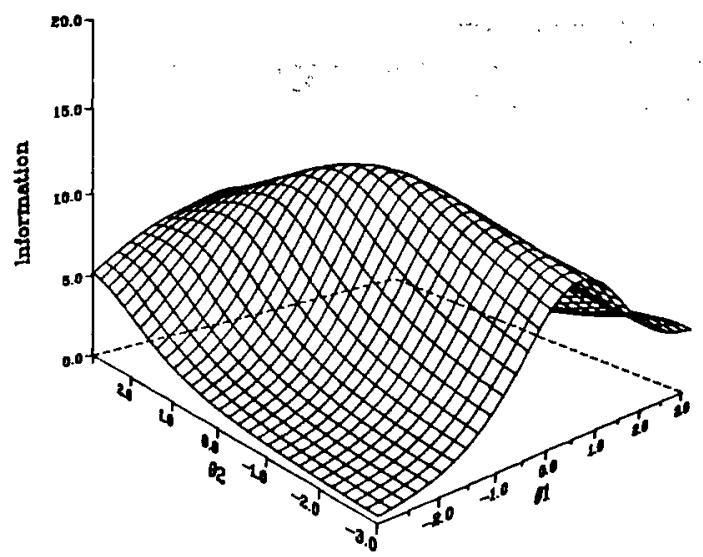

b. $45^{\circ}$

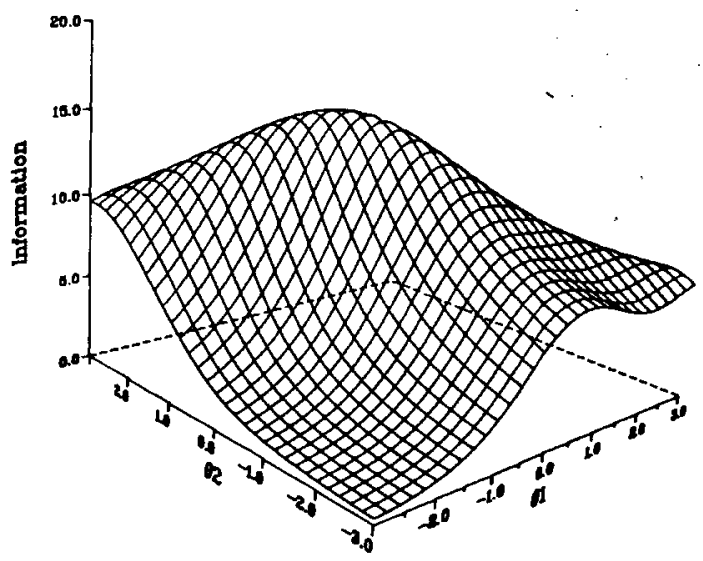

c. $90^{\circ}$

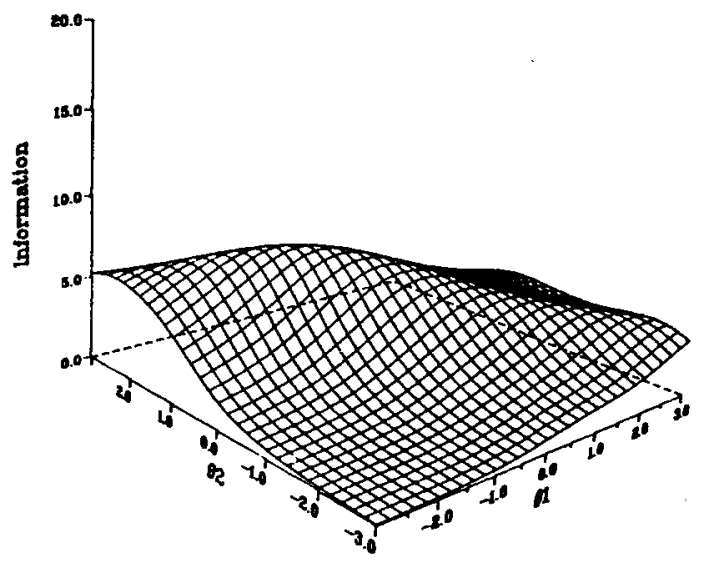




\section{Conclusions}

The multidimensional measures of item quality, item precision, and test precision given here provide a set of tools that can be used to gain a better understanding of the measurement process. Their use, in conjunction with appropriate multidimensional models, may lead to a more powerful class of measurement devices for most psychological and educational variables than those based on the relatively unrealistic unidimensional models.

\section{References}

American College Testing. (1982). The ACT Assessment Form 24B. Iowa City IA: American College Testing.

McKinley, R. L., \& Reckase, M. D. (1982). The use of the general Rasch model with multidimensional item response data (Research Rep. ONR 82-1). Iowa City IA: American College Testing.

McKinley, R. L., \& Reckase, M. D. (1983). MAXLOG: A computer program for the estimation of the parameters of a multidimensional logistic model. Behavior Research Methods and Instrumentation, 15, 389-390.

Reckase, M. D. (1985). The difficulty of test items that measure more than one ability. Applied Psychological Measurement, 9, 401-412.

Sympson, J. B. (1978). A model for testing with multidimensional items. In D. J. Weiss (Ed.), Proceedings of the 1977 Computerized Adaptive Testing Conference (pp. 82-98). Minneapolis: University of Minnesota, Department of Psychology, Computerized Adaptive Testing Laboratory.

Wingersky, M. S., Barton, M. A., \& Lord, F. M. (1982). LOGIST user's guide. Princeton NJ: Educational Testing Service.

\section{Acknowledgments}

This research was supported by Contract No. N00014-85-C-0241 with the Personnel and Training Research Programs of the Psychological Sciences Division of the Office of Naval Research.

\section{Author's Address}

Send requests for reprints or further information to Mark D. Reckase, American College Testing, P. O. Box 168, Iowa City IA 52243, U.S.A. 